\title{
ZEOLITAS DEL VALLE CENTRAL DE COSTA RICA Y SUS ALREDEDORES
}

\author{
Luis Alonso Zeledón \\ Apartado postal, 1280-2150 Moravia, San José, Costa Rica
}

(Recibido 6/6/04; Aceptado 7/2/05)

\begin{abstract}
Valle Central of Costa Rica and in the Siquirres region. All the zeolites are secondary minerals and crystallized in little veins and vesicles of the basalts and pyroclastic rocks of the La Cruz and Grifo Alto formations, belonging to the Aguacate Group and the alcaline igneous rocks of Guayacán. The most frequent species of zeolites are stibnite, chabasite, laumontite, mesolite/mordenite, thomsonite, and analcim. Natrolite only was found near to Siquirres.

Keywords: Zeolitas, outcrops, Valle Central, Costa Rica, especies

RESUMEN: Se describen 19 especies diferentes de zeolitas procedentes de 25 afloramientos en los alrededores del Valle Central de Costa Rica y en la región de Siquirres. Todas las zeolitas descritas son de origen secundario y se presentan en vetillas y vesículas de los basaltos y rocas piroclásticas de las formaciones La Cruz y Grifo Alto del Grupo Aguacate y en las rocas ígneas alcalinas de Guayacán. Las especies más frecuentes son estilbita, chabasita, laumontita, mesolita/mordenita, thomsonita y analcima. La natrolita solo se encontró en los alrededores de Siquirres.

Palabras clave: Zeolitas, afloramientos, Valle Central, Costa Rica, especies.
\end{abstract}

\section{INTRODUCCIÓN}

Las zeolitas, como familia mineral no se han estudiado detalladamente en nuestro país. En las publicaciones geológicas, por ejemplo, solamente se menciona su presencia en diversas rocas ígneas, pero no se presentan subdivisiones de este vasto grupo de minerales.

Químicamente, las zeolitas son alumosilicatos hidratados, que contienen porcentajes variables de calcio, sodio, bario, estroncio, potasio, manganeso y hierro. En la actualidad, la Asociación Internacional de Minerales (IMA) acepta 48 tipos diferentes de zeolitas, ordenados en subgrupos de acuerdo a su composición química y su hábito cristalográfico.

El nombre de zeolita fue dado por el mineralogista sueco Frederik Cronsted en 1756 y se deriva de las palabras griegas zein (hervir) y litos (piedra), debido a su comportamiento al ser calentadas con el soplete. Al principio del siglo XIX, René Just Haüy (1734 - 1822), mineralogista francés, introdujo en su Tratado de Mineralogía los primeros nombres a las diferentes especies de este grupo. Con la introducción de los rayos-X en 1920, se estudió su estructura cristalina y se empezó a caracterizar cada una de las especies (Dana \& Ford, 1973). 
Sus estructuras cristalinas consisten de tetraedros de aluminio, oxígeno y silicio y se diferencian de las estructuras de los demás minerales por poseer canales amplios que contienen moléculas de agua. Este hecho es el responsable de muchas de sus características y aplicaciones en la industria. Por ejemplo, al calentarse, el agua es eliminada paulatinamente, sin que el mineral sea destruido y posteriormente las zeolitas pueden absorber nuevamente el agua o también moléculas de otras sustancias. Las aplicaciones principales de las zeolitas son como cribas moleculares e intercambiadores de iones.

Zeolitas se presentan en todos los grupos de roca: ígneas, sedimentarias y metamórficas. Los cristales mejor desarrollados se encuentran en las vetillas y cavidades de las rocas ígneas. Los que se utilizan en la industria se extraen principalmente de tobas alteradas, donde las zeolitas se forman a partir del vidrio volcánico. Muchas de las zeolitas en las rocas ígneas y sedimentarias se forman por la reacción entre el agua y minerales, principalmente plagioclasa, nefelina y vidrio (Hay, 1978). Cuál especie de zeolita se forma, depende de la temperatura, presión y parámetros químicos (relación $\mathrm{Si} / \mathrm{Al}$, concentración de $\mathrm{Ca}^{2+}$, $\mathrm{Na}^{+} \mathrm{y} \mathrm{K}^{+}$, etc.

\section{HISTORIA DE LA NVESTIGACIÓN DE LAS ZEOLITAS EN COSTA RICA}

A mediados de 1982 vino a residir a Costa Rica Ronald Boyd, aficionado a los minerales, procedente de Canadá. Desde años atrás se dedicaba a la recolección de minerales en Nueva Escocia y Canadá. Empezó a recolectar minerales en nuestro país y a clasificar las zeolitas, dedicando especial atención a la zona sur del Valle Central.

Conoció a Rudy Tschernich, prestigioso investigador estadounidense en el campo de los minerales, con especial énfasis en las zeolitas, y a principios de 1986 le envió muestras para su clasificación. Tschernich publicó en 1992 "Zeolites of the World", un estudio amplio y detallado sobre este grupo de minerales y en el cual se incluyen las ocurrencias de zeolitas en nuestro país proporcionadas por Boyd.
En 1992 Boyd envió nuevamente algunas zeolitas desconocidas a Tschernich, procedentes de la zona de Pedernal de Puriscal, en especial una especie de hábito tetragonal bipiramidal. J. V. Smith, de la Universidad de Chicago, la define por difractometría de rayos-X como tschernichita, encontrándose entonces la segunda ocurrencia mundial para esta especie. Esto motivó a Tschernich a visitar Costa Rica en enero de 1993. Conjuntamente con Ronald Boyd visitaron diversos lugares y los resultados de sus observaciones aparecen en la revista Micro Probe, órgano del "Northwest Micro Mineral Group" de Estados Unidos. Se trata de las publicaciones "Zeolites of Costa Rica” (Tschernich, 1993a), un recuento de los lugares visitados y de las zeolitas encontradas y "Epistilbite and associated minerals from Pedernal, Costa Rica" (Tschernich, 1993b), en donde se definen las características cristalográficas de dos generaciones diferentes de esta zeolita y su paragénesis con otros minerales en este lugar. En el artículo "Preliminar report of the second occurencie of tschernichite" (Tschernich \& Boyd, 1993) definen a Pedernal de Puriscal como el segundo lugar a nivel mundial donde aparece esta zeolita escasa.

Posterior a estas publicaciones no se conocen nuevos aportes, pero el autor continuó con la exploración en otros lugares en los alrededores del Valle Central, extendiéndose a la zona de Siquirres.

\section{METODOLOGÍA}

Durante los doce últimos años, se realizaron giras de campo para la recolección de muestras, especialmente a las zonas de Acosta, Puriscal, Atenas, Palmares y Guayacán de Siquirres. En cada afloramiento, ya sea en canteras, espaldones del camino o cauces de ríos y quebradas, se extraen a golpes de mazo y cincel rocas que contienen cavidades rellenas de minerales secundarios. Se presta especial atención a las pequeñas vesículas con zeolitas, ya que en ellas se encuentra la mayor variedad, aunque no pueden ser observados a simple vista.

Se procede a clasificar las muestras en bruto y a partirlas con una prensa de puntas para evitar 
que la vibración las destruya y frecuentemente es necesario observarlas por la lupa estereoscópica. Muchas de las zeolitas están cubiertas por pátinas de óxidos de hierro y manganeso que se elimina sumergiéndolas en soluciones diluidas de ácido oxálico y acético, neutralizándolas posteriormente con soluciones alcalinas.

Las muestras más frágiles se limpian sumergiéndolas por algunos minutos en una cubeta ultrasónica. Algunas muestras son tan frágiles que no se pueden limpiar y tienen que dejarse como se les encuentra. Las muestras más grandes y fuertes se lavan con agua y jabón y un cepillo de cerdas suaves.

Terminado el proceso de limpieza se procede a su montaje y etiquetado final. Para las muestras microscópicas se usa una lupa estereoscópica de 30 aumentos. Las fotografías fueron hechas con una cámara digital Nikon Coolpix de 2,5 mega píxeles y un aumento digital de $3 x$.

\section{AFLORAMIENTOS CON ZEOLITAS}

\section{La Cruz de Acosta}

El afloramiento se encuentra a 200 metros del poblado de La Cruz de Acosta, camino a Guaitil (coordenadas Lambert 513,2E - 197,5N, hoja Carai-gres; Fig. 1). Se caracteriza por un talud de unos 300 metros de largo, compuesto principalmente por coladas de basaltos con un espesor promedio de $20 \mathrm{~m}$ de la Formación La Cruz (Denyer \& Arias, 1991).

Las zeolitas se encuentran rellenando vesículas de varios centímetros de diámetro. Son de color blanco rosado, algunas con hábito fibroso, característico de la mordenita/ mesolita. En este lugar se han encontrado cristales de analcima hasta de $7 \mathrm{~cm}$ de diámetro. Las zeolitas se encuentran también dentro de vetillas de varios centímetros de espesor y rellenando diaclasas.

En esta localidad se han encontrado laumontita, heulandita, analcima, thomsonita, estilbita, chabasita, mordenita/ mesolita?, levinita y cowlesita. Todas ellas asociadas con calcita, calcopirita y a algunos minerales aún no identificados.

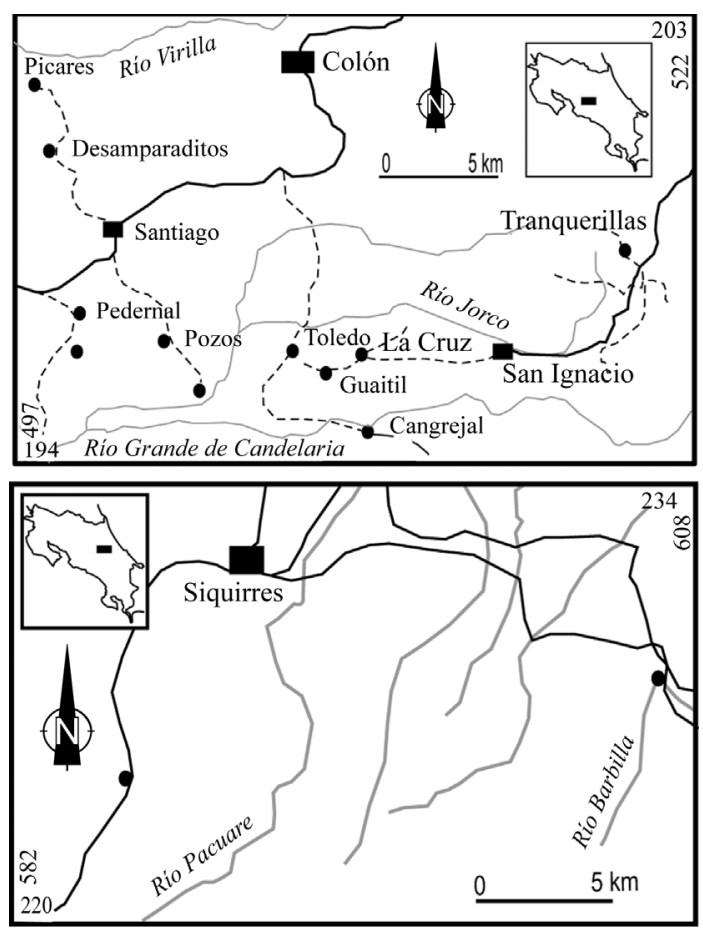

Fig. 1: Mapas de ubicación de los afloramientos.

En otro lugar cercano (515,3E -197,2 N), en el camino que lleva a Resbalón de Acosta, se encuentran en el piso del camino y en un pequeño talud, nódulos blancos de varios centímetros de diámetro rellenados con mordenita/mesolita y heulandita.

\section{Guaitil de Acosta}

Cerca de Guaitil, en un antiguo tajo localizado en 511,5E - 197,2N (hoja Caraigres; Fig. 1) afloran basaltos de la Formación La Cruz. En la parte superior se encuentran vetillas de varios centímetros de espesor, de color blanco, conformadas por cuarzo y zeolitas. El cuarzo es masivo, blanco lechoso y en los espacios abiertos se le encuentra en prismas transparentes y translúcidos, algunos con tinte amatistino. Posiblemente, cristales microscópicos de cristobalita con la forma de "granos de arroz", asociada con estilbita y muy escasamente con yugawaralita(?) forman una costra de recubrimiento. 
En la parte basal del tajo, el basalto está muy meteorizado y desmenuzable, con nódulos de varios milímetros a pocos centímetros de diámetro rellenos de cuarzo, estilbita, heulandita y mordenita/mesolita y en cantidades variables de chabasita, estilbita, laumontita, epistilbita y cuarzo, todos con tonos blanco lechosos.

\section{Bajo Calvo de Acosta}

Aproximadamente a unos 700 metros de esta localidad, en 511E - 198N (hoja Caraigres), en el talud del camino, afloran basaltos de la Formación La Cruz con vesículas rellenas de chabasita, heulandita, laumontita, levinita, filipsita, mesolita, analcima y gonardita.

\section{Toledo de Acosta}

En el camino a Pozos de Puriscal, en 510E - 198,4N (hoja Caraigres), en el piso y taludes, se observan gran cantidad de vetillas blancas rellenas de estilbita, filipsita y laumontita. Todas tienen un tono blanco lechoso y un tamaño de pocos milímetros.

\section{Quebrada Limonal}

A poca distancia de la confluencia de la quebrada Limonal con el río Candelaria (513,4E 194,6N, hoja Caraigres) se encuentran cavidades de pocos milímetros de diámetro rellenas de laumontita, asociada con malaquita, calcopirita y cerusita.

\section{Río Viejo de Puriscal}

Cerca de San Rafael, en la unión de los ríos Marín y Viejo de Puriscal (506,5E 201,8N; hoja Río Grande) se encuentra una zona de alteración hidrotermal de color amarillenta con analcima, chabasita, thomsonita, filipsita y estilbita.

\section{Quebrada Grande de Desamparaditos de Puriscal}

Aguas arriba del puente, en 498E - 207,5N (hoja Río Grande), afloran basaltos de la Formación Grifo Alto con vesículas desde varios milímetros a varios centímetros de diámetro rellenas de analcima, chabasita, thomsonita, filipsita, estilbita, heulandita, levinita, mordenita/mesolita?, cowlesita, asociadas con calcita.

\section{Pedernal de Puriscal}

A $2 \mathrm{~km}$ al NE de Pedernal, en 501E 200,5N (hoja Candelaria; Fig. 1), en los paredones de un conglomerado basáltico de los depósitos parálicos de la Formación La Cruz (Denyer \& Arias, 1991) quedan expuestas gran cantidad de vetillas y de vesículas huecas que contienen las siguientes zeolitas: Estilbita, chabasita, heulandita, epistilbita, levinita, mordenita/mesolita, erionita/o-ffretita?, cowlesita, thomsonita, tschernichita, filipsita, goosecreekita(?) y garronita. Están asociadas con okenita, apofilita, calcita y todorokita.

El orden general de cristalización observada es: Arcilla $>$ calcita $>$ tschernichita $>$ epistilbita-1 $>$ mordenita $>$ heulandita $>$ epistilbita- $2>$ estilbita $>$ escolecita? $>$ cowlesita $>$ levinita $>$ offretita $>$ thomsonita $>$ mesolita? $>$ okenita $>$ chabasita (Tschernich, 1993b)

\section{Bajo Chacones de Puriscal}

En un camino secundario al sureste de Pedernal, en 501E - 198N (hoja Candelaria) afloran basaltos meteorizados de la Formación La Cruz que contienen vetillas y vesículas blanquecinas rellenas de mordenita/escolecita, estilbita, laumontita, asociadas con cuarzo y calcita. La secuencia de cristalización observada es: Mordenita/mesolita $>$ cuarzo $>$ estilbita $>$ laumontita. 


\section{Pozos de Puriscal}

En un camino en Pozos de Puriscal (504E 198,4N, hoja Candelaria) se presentan dentro de los basaltos de la Formación La Cruz vesículas y vetillas de color blanco con mesolita/mordenita? y estilbita.

\section{Río Jesús María de Picagres, Puriscal}

En las cercanías de la población de Picagres, en el camino que se intersecta con el río Jesús María, se encuentran rocas con vesículas blancas rellenas de chabasita con formas romboédricas y placolita en varios tipos de formas, asociadas con heulandita.

\section{Santa Rosa de Puriscal}

En 492E - 188,2N (hoja Candelaria), se presenta chabasita microscópica de tono anaranjado en un talud del camino.

\section{Río Turrubares}

En 490E - 202,5N (hoja Río Grande), cerca de la confluencia con el río San José, se encuentran vetillas y nódulos compactos de varios centímetros de diámetro y de tono amarillento, rellenos de estilbita.

\section{Tranquerillas}

En los paredones del camino al NW de Tranquerillas (525E - 199N, hoja Caraigres, Fig. 1) se encuentran vetillas blancas con chabasita $y$ laumontita.

\section{Puente La Garita}

En la cantera cerca del puente La Garita, en 498,7E - 218,8N (hoja Río Grande) afloran basaltos y brechas de la Formación Grifo Alto (Denyer \& Arias, 1991) con gran cantidad de vetillas blancas rellenas de las siguientes zeolitas: Thomsonita, chabasita romboédrica y variedad placolita, mesolita/mordenita?, asociada con calcita en cristales de varios centímetros de tamaño y de color blanco y amarillento, transparentes, con poca apofilita y girolita (Tschernich, 1993a).

\section{Cerro Crisanto, Palmares}

En un tajo localizado en el cerro Crisanto (492E - 225N, hoja Naranjo) se encuentran chabasita y thomsonita asociadas con calcita.

\section{Tajo Dantas}

En la línea del ferrocarril, en 484,5E - 211,8N (hoja Río Grande), se encuentra mesolita/escolecita asociada con cuarzo y calcita rellenando vetillas en las rocas de la Formación Grifo Alto.

\section{San Ramón de Alajuela}

En la fila entre el río Jesús María y la quebrada Obispo (aproximadamente 476E - 221N; hoja Miramar) se presentan vetillas de varios centímetros de espesor con estilbita y laumontita, asociadas con cuarzo.

\section{Tajo Cóncavas}

En la cantera localizada 2,5 $\mathrm{km}$ al oeste de Paraíso de Cartago (2548,3E - 202,3N; hoja Istarú) se encuentran dentro de una secuencia basáltica, laumontita en grandes masas y mordenita/escolecita en cristales aciculares de tonos café claro, asociada con calcita y minerales de cobre, tales como calcopirita, bornita, calcosina y malaquita.

\section{Cerro Estaquero}

En la Carretera Interamericana Sur, en 489E - 395N (hoja Vueltas), está expuesto en los cortes 
del cerro, vetillas de varios centímetros de espesor que contienen heulandita y estilbita en cristales microscópicos de tono lechoso.

\section{Estanquillo}

Cerca de Desmonte de Atenas, en las coordenadas 486E - 219,5N (hoja Río Grande), se encuentran chabasita, thomsonita y filipsita en vetillas blancas.

\section{Alto de Guayacán}

Tournon (1984) describe una secuencia de basaltos alcalinos y teschenitas con analcima intersticial que afloran a lo largo de la carretera Turrialba - Siquirres y que contienen vesículas rellenas de zeolitas.

En el Alto de Guayacán, en 586,5E - 225,2N (hoja Bonilla; Fig. 1), en el camino que lleva a la quebrada Quebradón, se encuentran las siguientes zeolitas: Chabasita, natrolita/paranatrolita, levinita, erionita/offretita?, filipsita, en algunas ocasiones asociadas con calcita.

\section{Calle Petróleo}

En 584,5E - 224,3N (hoja Bonilla), en el camino que sirve de acceso para el oleoducto, se encuentran bloques de basaltos con chabasita, levinita y erionita/offretita?.

\section{Río Barbilla}

En el puente sobre la carretera que conduce a Limón (Fig. 1), en las rocas arrastradas por la corriente se encuentran fragmentos de basaltos vesiculares que contienen las siguientes zeolitas: Chabasita romboédrica y variedad placolita (Fig. 2) analcima, thomsonita, filipsita, gismodina, levinita, erionita/offretita? y natrolita/mesolita?, asociadas con cuarzo, calcita y prehnita.

\section{Río Madre de Dios}

En la carretera después de Siquirres, sobre el puente aguas arriba se encuentra chabasita de la variedad placolita.
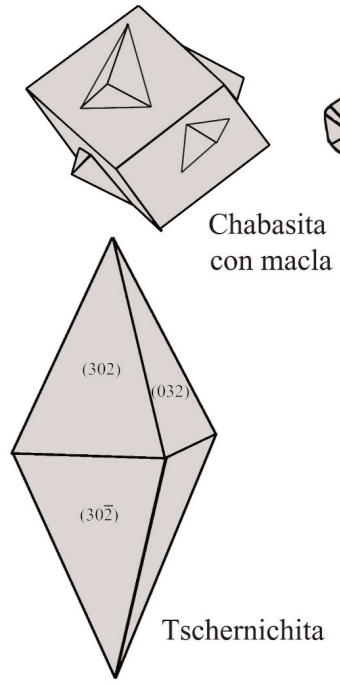

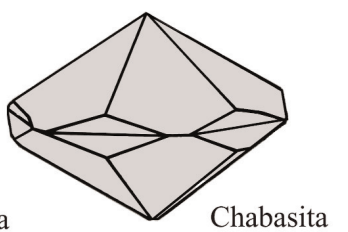

var. placolita

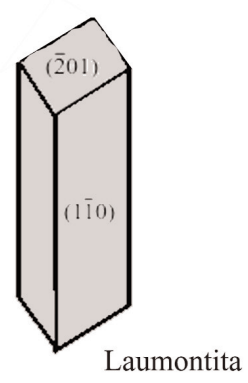

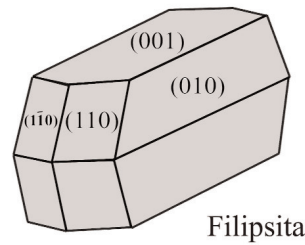
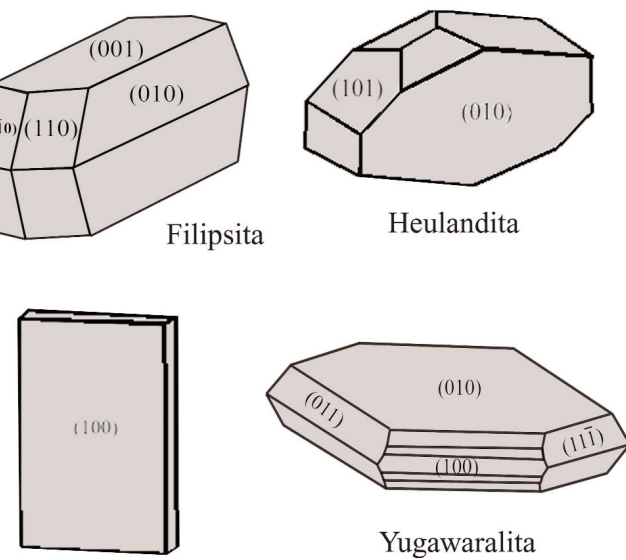

Yugawaralita

Thomsonita

Fig. 2: Formas cristalinas características de algunas especies de zeolita. 


\section{DESCRIPCIÓN DE LAS ESPECIES DE ZEOLITAS}

\author{
Analcima $\mathrm{Na}\left[\mathrm{AlSi}_{2} \mathrm{O}_{6}\right] * \mathrm{H}_{2} \mathrm{O}$
}

Cristaliza en el sistema cúbico y frecuentemente presenta cristales en forma trapezoédrica o dodecaédrica, agrupados en las paredes de vesículas (Fig. 3: 1). Son transparentes o translúcidos con tonos amarillentos o tenuemente lechosos.

Está cubierta por oxidaciones verdosas y rojas y se presenta junto a chabasita (en Desamparaditos) o de natrolita y chabasita (en el río Barbilla). En La Cruz de Acosta rellena vetas y geodas de varios centímetros de diámetro, con cristales de tono lechoso rosado de hasta $8 \mathrm{~cm}$, asociada con thomsonita.

\section{Chabasita $\mathrm{Ca}\left[\mathrm{Al}_{2} \mathrm{Si}_{4} \mathrm{O}_{12}\right] * 6 \mathrm{H}_{2} \mathrm{O}$}

Cristaliza en el sistema trigonal pero se presenta por lo general en cristales de hábito seudocúbico (Fig. 3: 2) y con maclas de compenetración (Fig. 2). Presenta estriaciones en las caras evidenciándose la disposición de la compenetración de los individuos maclados. En la variedad placolita (Fig. 3: 3), varios individuos se entrecruzan de tal forma que producen estructuras tabulares seudohexagonales en la forma de platillos voladores (Fig. 2). Existe una mezcla química entre la chabasita y la gmelinita, la primera rica en calcio, la otra en sodio, por lo que se confunden en las fases intermedias y producen clasificaciones inciertas, especialmente en la variedad placolita.

Se la encuentra en cristales transparentes y translúcidos, rellenando cavidades de pocos milímetros de diámetro, asociada con calcita y heulandita, mordenita / mesolita (Cruz de Acosta); recubriendo vetillas asociada con thomsonita y calcita (tajo la Garita); dentro de drusas de cuarzo en cristales de tonos blanco lechoso o nacarados, asociada con epistilbita, heulandita, laumontita (Guaitil de Acosta); en cristales transparentes de $2-3 \mathrm{~cm}$ asociada con thomsonita (cerro Crisanto).
La variedad placolita rellena cavidades mayores de $10 \mathrm{~cm}$ de diámetro, es transparente a lechosa y asociada con filipsita en Desamparaditos de Puriscal. En las paredes de vesículas también está asociada con prehnita (río Barbilla) y como recubrimiento masivo de vetillas de color blanco amarillento (tajo la Garita).

\section{Cowlesita $\mathrm{Ca}\left[\mathrm{Al}_{2} \mathrm{Si}_{3} \mathrm{O}_{10}\right] * 5-6 \mathrm{H}_{2} \mathrm{O}$}

Cristaliza en el sistema rómbico y se encuentra en cristales con forma de navajas muy pequeños, tapizando en su totalidad cavidades de 3 a 8 milímetros de diámetro (Fig. 3: 4). Es muy frágil, de un color blanco perlado con brillo nacarado, asociada con levinita en La Cruz de Acosta y en Pedernal de Puriscal.

\section{Epistilbita $\mathrm{Ca}\left[\mathrm{Al}_{2} \mathrm{Si}_{6} \mathrm{O}_{16}\right] * 5 \mathrm{H}_{2} \mathrm{O}$}

Cristaliza en el sistema monoclínico y se caracteriza por formar prismas alargados pseudotabulares, producto del maclado de dos individuos que terminan en ángulos afilados (Fig. 3: 5 y 6). Por lo general se le encuentra rellenando cavidades de pocos milímetros de diámetro. En Pedernal de Puriscal fue reportado por Tschernich (1993b), quien observó dos generaciones distintas. Una con hábito de prismas cortos, transparentes y translúcidos, rellenando la totalidad de las cavidades, la otra en prismas con estriaciones verticales, asociada con levinita. En Guaitil de Acosta se le encuentra dentro de drusas de cuarzo, en cristales de 3 a 6 mm de largo, de color blanco lechosos, con brillo nacarado o sucio debido a pátinas de óxidos de manganeso y hierro, asociada con chabasita, heulandita, laumontita y cuarzo.

$$
\begin{aligned}
& \text { Erionita }\left(\mathrm{Na}_{2}, \mathrm{~K}_{2}, \mathrm{Ca}\right)\left[\mathrm{Al}_{4} \mathrm{Si}_{4} \mathrm{O}_{36}\right] * 15 \mathrm{H}_{2} \mathrm{O} \\
& \text { Offretita }\left(\mathrm{K}_{2}, \mathrm{Ca}\right){ }_{5}\left[\mathrm{Al}_{5} \mathrm{Si}_{13} \mathrm{O}_{36}\right]_{2} * 30 \mathrm{H}_{2} \mathrm{O}
\end{aligned}
$$

Solo es posible diferenciarlas por difracción de rayos-x. Ambos minerales cristalizan en el sistema hexagonal y se presentan como agregados aciculares muy cortos, formando un recubrimiento 

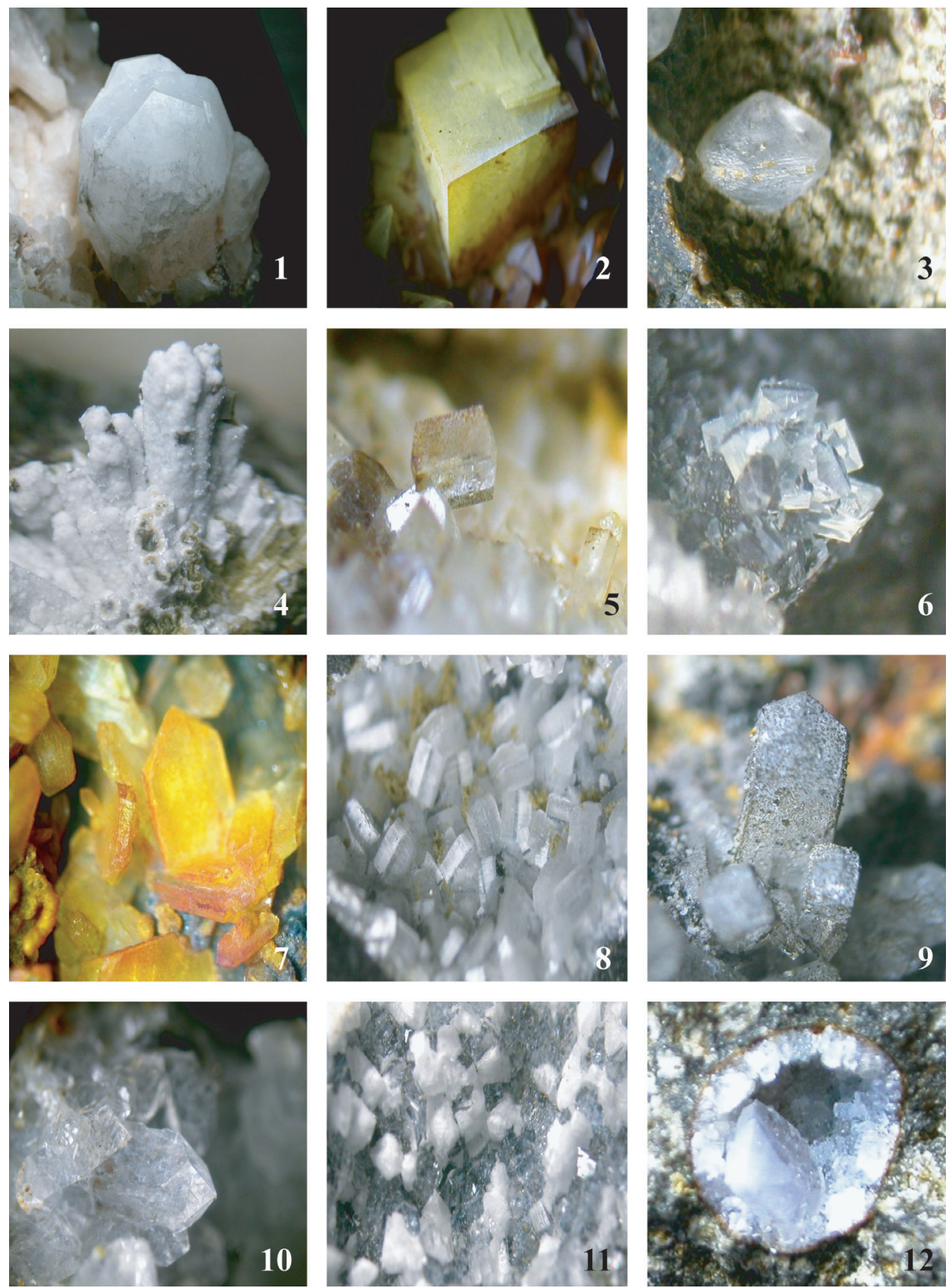

Fig. 3: Microfotografias de zeolitas de Costa Rica:

1.- Analcima sobre estilbita (30x), La Cruz de Acosta

2.- Chabasita (30x), Guaitil de Acosta

3.- Chabasita, var. Placolita (30x), río Barbilla. Siquirres

4.- Cowlesita (35x), La Cruz de Acosta

5.- Epistilbita (40x), La Cruz de Acosta

6.- Epistilbita, cristales recubiertos por erionita (30x),
Pedernal de Puriscal

7.- Estilbita (30x), Pedernal de Puriscal

8.- Erionita-Offretita(?) (30x), Río Barbilla, Siquirres

9.- Filipsita (30x), Desamparaditos, Puriscal

10.- Filipsita (30x), Desamparaditos, Puriscal

11.- Gismondina, Río Barbilla, Siquirres

12.- Goosecrekita (30x) Pedernal de Puriscal 
fino de cristales de tono lechosos sobre levinita y chabasita (en Pedernal de Puriscal). En Guayacán y río Barbilla de Siquirres cristales aciculares divergentes, sedosos, blanco grisáceos, rellenan cavidades de varios centímetros de diámetro, cristalizando sobre levinita y chabasita.

Estilbita $\left(\mathrm{Ca}, \mathrm{Na}_{2}\right)\left[\mathrm{Al}_{2} \mathrm{Si}_{7} \mathrm{O}_{18}\right] * 7 \mathrm{H}_{2} \mathrm{O}$

Este grupo está conformado por las especies estilbita, estellarita y barrierita, cuya forma es idéntica, pero que presentan variaciones en las cantidades de sodio y calcio. Se ha establecido el nombre general de estilbita para referirse a estas especies que cristalizan en el sistema monoclínico, seudorómbico (Fig. 3: 7). Por lo general se le encuentra en cristales tabulares, agrupados en haces, generalmente en contacto por su cara (010).

En nuestro país se encuentran desde tamaños microscópicos hasta individuos de varios centímetros de largo. Los cristales pequeños tienden a ser transparentes y translúcidos, los más grandes tienen tonos amarillentos y blancos perlados. En Pedernal rellenan vesículas de pocos milímetros de diámetro. Presentan maclas de contacto formando abanicos y están asociados a heulandita y chabasita. También rellenan vetillas de color blanco perlado, con manchas de óxidos de manganeso y hierro. En Guaitil se presenta en nódulos de cuarzo o en vetillas de cuarzo lechoso asociado con cristobalita. En Bajo Chacones de Puriscal aparece como cristales de gran tamaño, asociada con laumontita que rellenan vetas y en Pozos de Puriscal está asociada con mordenita / escolecita, con tonos anaranjados y recubiertas por óxidos de hierro y arcillas.

Filipsita $(1 / 2 \mathrm{Ca}, \mathrm{Na}, \mathrm{K})_{5}\left[\mathrm{Al}_{5} \mathrm{Si}_{11} \mathrm{O}_{32}\right] * 10 \mathrm{H}_{2} \mathrm{O}$

Cristaliza en el sistema monoclínico y se caracteriza por formar prismas aplastados de cuatro lados, con punta terminada en cuatro caras paralelas en pares, ligeramente levantadas en un punto central (Fig. 2). A menudo forma maclas con pequeñas estriaciones en sus caras, que aparentan una simetría superior (Fig. 3: 9 y 10). Ya sea como cristales individuales o formando maclas, cubren en forma desordenada o como agregados pequeños divergentes, las paredes de las cavidades.
En Desamparaditos de Puriscal son comunes cristales de 5 a $8 \mathrm{~mm}$ de largo, de tono blanco lechoso, con oxidaciones oscuras, rellenando cavidades, asociada con calcita, thomsonita y analcima. En Guayacán y río Barbilla se presenta en forma de agregados esféricos de prismas transparentes y translúcidos, rellenando cavidades de varios milímetros de diámetro.

Garronita $\mathrm{Na}_{2} \mathrm{Ca}_{5}\left[\mathrm{Al}_{3} \mathrm{Si}_{5} \mathrm{O}_{16}\right]_{4} * 27 \mathrm{H}_{2} \mathrm{O}$

Cristaliza en el sistema tetragonal y se presenta en masas fibrosas, seudoésféricas, de color blanco perlado en cavidades de pocos milímetros en Pedernal de Puriscal (Tschernich, 1993).

\section{Gismondina $\mathrm{Ca}\left[\mathrm{Al}_{2} \mathrm{Si}_{2} \mathrm{O}_{8}\right] * 4 \mathrm{H}_{2} \mathrm{O}$}

Cristaliza en el sistema monoclínico, pseudo-tetragonal y se la ha encontrado únicamente en el río Barbilla, donde se presenta como un recubrimiento sobre chabasita, con color blanco lechoso y en forma de cristales piramidales cortos de cuatro lados.

\section{Gonardita $\mathrm{Na}_{2} \mathrm{Ca}\left[\mathrm{Al}_{4} \mathrm{Si}_{6} \mathrm{O}_{20}\right] * 7 \mathrm{H}_{2} \mathrm{O}$}

Cristaliza en el sistema tetragonal. Tschernich \& Boyd (1993) la reportan de Bajo Calvo de Acosta, donde se presenta, junto con filipsita y chabasita, en forma de agregados esféricos blancos ahumados de hasta $15 \mathrm{~mm}$ de diámetro.

\section{Goosecrekita $\mathrm{Ca}\left[\mathrm{Al}_{2} \mathrm{Si}_{6} \mathrm{O}_{16}\right] * 5 \mathrm{H}_{2} \mathrm{O}$}

$\mathrm{Su}$ presencia no es muy segura y es una especie escasa. Cristaliza en el sistema monoclínico y solo se le ha encontrado en Pedernal de Puriscal en pocos cristales solitarios en cavidades de pocos milímetros de diámetro (Fig. 3: 12). Tiene un hábito tabular, ligeramente recurvados en la base y es de color blanco grisáceo a tenuemente celeste.

\section{Heulandita $\mathrm{Ca}\left[\mathrm{Al}_{2} \mathrm{Si}_{2} \mathrm{O}_{18}\right] * 6 \mathrm{H}_{2} \mathrm{O}$}

Cristaliza en el sistema monoclínico y se presenta, por lo general, en cristales de hábito 
tabular (Fig. 2), con caras trapezoédricas (Fig. 4: 13 y 14). Es transparente o translucida, con tonos blanco lechoso y amarillentos. Forma agregados con contactos por su cara (010), dando la apariencia de prismas alargados. Rellena vesículas junto con chabasita, calcita, epistilbita y mordenita (Pedernal), en tonos lechosos con cuarzo, epistilbita y laumontita (Guaitil de Acosta) o rellena geodas de varios centímetros de diámetro, con cristales de tonos blanco, rosados y perlados (La Cruz).

\section{Laumontita $\mathrm{Ca}\left[\mathrm{AlSi}_{2} \mathrm{O}_{6}\right]_{2} * 4 \mathrm{H}_{2} \mathrm{O}$}

Cristaliza en el sistema monoclínico, generalmente en forma de prismas poco alargados de cuatro lados y terminados en punta (Fig. 4: $15 \mathrm{y}$ 16; Fig. 2). También forma maclas alargadas de contacto con forma de punta de flecha con ligeras estriaciones en las caras largas del cristal. Generalmente es de color blanco perlado, con pátinas de arcillas u óxidos. Es una zeolita muy frágil, que se rompe fácilmente, especialmente cuando se deshidrata.

Se le encuentra en cristales de 3 a $6 \mathrm{~mm}$, asociados con minerales de cobre (Limonal de Acosta), en cristales prismáticos de varios centímetros de largo, asociada con estilbita y en masas de múltiples individuos, rellenando grietas (Bajo Chacones de Puriscal). En Cóncavas de Cartago se presenta en grandes masas blanquecinas de varios kilos de peso, asociada con calcita y a minerales de cobre como calcopirita, calcosina y malaquita. En la quebrada Obispo en San Ramón de Alajuela se formó sobre cuarzo y estilbita.

\section{Levinita $\left(\mathrm{Ca}, \mathrm{Na}_{2}, \mathrm{~K}_{2}\right)\left[\mathrm{Al}_{2} \mathrm{Si}_{4} \mathrm{O}_{12}\right] * 6 \mathrm{H}_{2} \mathrm{O}$}

Cristaliza en el sistema trigonal y se le encuentra casi siempre en tamaños microscópicos, en placas hexagonales, también en forma de maclas, unidas por su cara más plana, dando una apariencia más gruesa a las placas, con bordes biselados y más anchos hacia la base del contacto, formando de maclas (Fig. 4: 17 y 18).

En Pedernal de Puriscal se presenta en placas de 3 a 5 milímetros de diámetro, transparentes y translúcidas, con tonos mates y lechosos, en grupos de cristales paralelos rellenando parte de cavidades, asociada con epistilbita y offretita/ erionita (?) que los recubre. En La Cruz de Acosta forma maclas de contacto, anchas y biseladas. En Guayacán y Barbilla de Siquirres se la encuentra como placas muy delgadas de color oscuro, recubiertas a ambos lados por fibras sedosas de color gris blanquecino de erionita/offretita(?), formando un emparedado. La levinita es una especie muy difundida en la Cruz de Acosta, Pedernal de Puriscal y en Guayacán, donde forma maclas complejos según su cara (0001) y con hábito esférico.

Mordenita $\left(\mathrm{Na}_{2}, \mathrm{~K}_{2}, \mathrm{Ca}\right)\left[\mathrm{AlSi}_{5} \mathrm{O}_{12}\right]_{2} * 6 \mathrm{H}_{2} \mathrm{O}$
Escolecita $\mathrm{Ca}\left[\mathrm{Al}_{2} \mathrm{Si}_{3} \mathrm{O}_{10}\right]_{2} * 3 \mathrm{H}_{2} \mathrm{O}$

Son zeolitas que cristalizan en el sistema rómbico y monoclínico y en macroscopía es imposible diferenciarlas, por lo cual se describen juntas. Se presentan en forma fibrosa y acicular; desde cristales aislados a agregados divergentes como abanicos y ramilletes de agujas (Fig. 4: 19). Son transparentes a translúcidos, de color blanco lechoso y café claro, muchas veces sucios por oxidaciones y arcillas. Están acompañadas con frecuencia por otras zeolitas y minerales accesorios.

En Desamparaditos de Puriscal se les encuentra como agregados aciculares, acompañando a thomsonita y analcima. En La Cruz de Acosta forman masas sobre thomsonita, analcima, estilbita y chabasita. En Guaitil se presentan como agujas que recubren cuarzo, asociadas con estilbita y chabasita. En Pozos de Puriscal acompañan a la estilbita como agregados aciculares con tonos anaranjados y en Pedernal de Puriscal están asociadas con muchas zeolitas y forman agujas atravesando cristales de estilbita.

$$
\begin{aligned}
& \text { Mesolita } \mathrm{Na}_{2} \mathrm{Ca}\left[\mathrm{Al}_{2} \mathrm{Si}_{3} \mathrm{O}_{10}\right]_{3} * 8 \mathrm{H}_{2} \mathrm{O} \\
& \text { Natrolita } \mathrm{Na}_{2}\left[\mathrm{Al}_{2} \mathrm{Si}_{3} \mathrm{O}_{10}\right] * 2 \mathrm{H}_{2} \mathrm{O}
\end{aligned}
$$

Cristalizan en el sistema rómbico y monoclinico, al igual que los tres minerales anteriores son zeolitas fibrosas (Fig. 4: 20 y 21). Son especies poco frecuente en Costa Rica. Se caracterizan por formar prismas alargados en forma de 

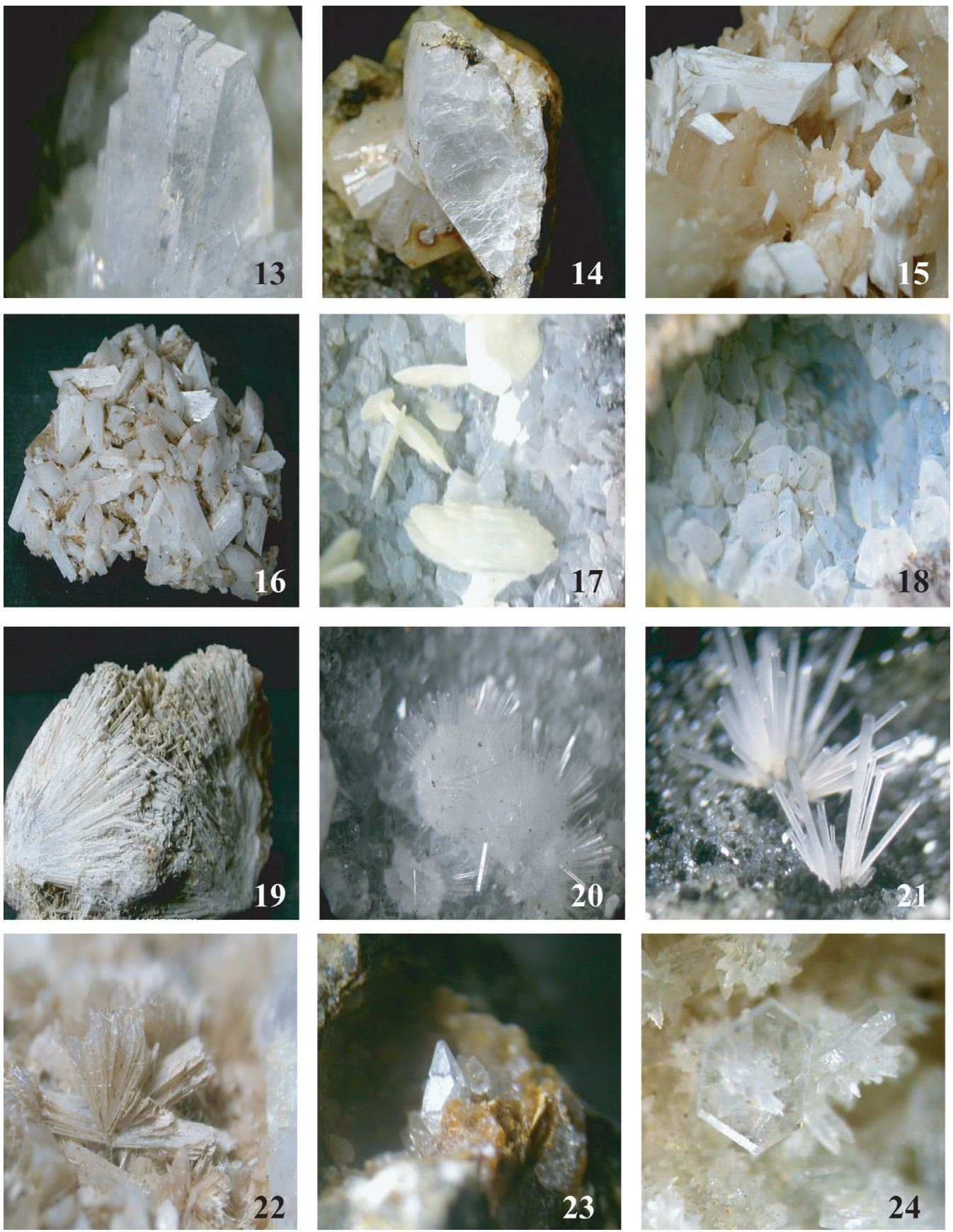

Fig. 4: Microfotografias de zeolitas de Costa Rica:

13.- Heulandita (30x), Guaitil de Acosta

14.- Heulandita (30x), Guaitil de Acosta

15.- Laumontita (3x), Bajo Chacones de Puriscal

16.- Laumontita (2x), Bajo Chacones de Puriscal

17.- Levinita sobre epistilbita (30x), Pedernal de Puriscal

18.- Levinita (30x), La Cruz de Acosta
19.- Mordenita (2x), Pozos de Puriscal

20.- Mesolita sobre thomsonita (40x), Desamparaditos de Puriscal

21.- Natrolita (40x) Guayacán de Siquirres 22.- Thomsonita (45x), La Cruz de Acosta 23.- Tschernichita (40x), Pedernal de Puriscal 24.- Yugawaralita (35x), Guaitil de Acosta 
agujas de cuatro lados, terminando en una pirámide corta de cuatro lados o en corte recto.

En el río Barbilla es transparente a translúci$\mathrm{da}$, frecuentemente en grupos radiales y asociada con thomsonita y analcima. En Guayacán de Siquirres se presenta dentro de cavidades como un agregado radial de pocos milímetros de largo. En Desamparaditos es frecuente asociada a otras zeolitas y minerales.

Thomsonita $\mathrm{NaCa}\left[\mathrm{Al}_{5} \mathrm{Si}_{5} \mathrm{O}_{20}\right] * 6 \mathrm{H}_{2} \mathrm{O}$

Cristaliza en el sistema rómbico y se la encuentra conformando agrupaciones radiales y seudoesféricas de cristales en forma de navajas delgadas (Fig. 4: 22, Fig. 2), de transparentes a lechosas, por lo general unidas a la cara más plana en la base y ligeramente divergentes hacia afuera. En la mayoría de las localidades, su tamaño es microscópico; cristales grandes son raros.

En La Garita se presenta en agregados radiales, transparentes y translúcidos de color amarillento y anaranjado, dispuestos sobre chabasita y calcita. En Desamparaditos, agregados radiales rellenan cavidades en asociación con mesolita(?) y chabasita. En Pedernal de Puriscal se la encuentra en grietas en forma de navajas de tono café claro recubierta por cristales aciculares de mesolita(?). En La Cruz de Acosta se presenta en forma de navajas rellenando los espacios entre cristales de analcima de 2 a $4 \mathrm{~cm}$ de tamaño. En el río Barbilla las navajas se reúnen como agregados transparentes asociadas con mesolita(?) y rellenan vesículas de pocos milímetros de diámetro.

\section{Tschernichita $\left(\mathrm{Na}_{2}, \mathrm{Ca}\right)\left[\mathrm{Al}_{2} \mathrm{Si}_{6} \mathrm{O}_{16}\right] * 4-8 \mathrm{H}_{2} \mathrm{O}$}

Cristaliza en el sistema tetragonal. Se le encuentra dentro de cavidades de pocos milímetros de diámetro en forma de cristales bipiramidales tetragonales (Fig. 2), terminados en punta o truncados, con estriaciones horizontales tenues (Fig. 4: 23). Es transparente o translucida, incolora o con tonos de amarillo limón.

Es un mineral escaso, descubierto en nuestro país por Ronald Boyd. Pedernal de Puriscal es la segunda ocurrencia a nivel mundial de esta zeolita. Su tamaño varía entre 0,1 y $0,3 \mathrm{~mm}$ y solo está asociada con la levinita y epistilbita, los cuales cristalizaron después de la tschernichita; sin embargo, en vesículas vecinas se pueden presentar muchas de las otras zeolitas (Tschernich \& Boyd, 1993).

Yugawaralita $\mathrm{Ca}\left[\mathrm{Al}_{2} \mathrm{Si}_{6} \mathrm{O}_{16}\right] * 4 \mathrm{H}_{2} \mathrm{O}$

Su presencia en Costa Rica no está confirmada. Cristaliza en el sistema monoclínico y se le encuentra en forma tabular (Fig. 2), elongada hacia los polos, terminada en punta angulada y con estriaciones verticales apenas perceptibles en sus caras laterales.

Solo se presenta en Guaitil de Acosta en geodas y vetillas sobre cuarzo tipo "grano de arroz" (probablemente cristobalita de tono lechoso) en forma de cristales aislados y transparentes y, también, implantado con cristales de estilbita, de la cual solamente por difracción de rayos-x se puede diferenciar.

\section{DESCRIPCIÓN DE ALGUNOS DE LOS MINERALES ASOCIADOS}

Apofilita $\mathrm{KCa}_{4} \mathrm{~F}\left[\mathrm{Si}_{4} \mathrm{O}_{10}\right]_{2} * 8 \mathrm{H}_{2} \mathrm{O}$

Se caracteriza por prismas piramidales de cuatro lados y punta truncada. Es transparente, translúcida, con tonos lechosos y acompaña a las zeolitas en Pedernal de Puriscal y en río Barbilla en Siquirres, con menos frecuencia en Desamparaditos de Puriscal.

\section{Azurita $\mathrm{Cu}_{3}\left[(\mathrm{OH}) \mathrm{CO}_{3}\right]_{2}$}

Mineral de cobre de color azul característico, que tapiza cavidades y está asociada con laumontita en la quebrada Limonar de Acosta.

\section{Cuarzo $\mathrm{SiO}_{2}$}

Es muy frecuente en cristales transparentes y traslúcidos, especialmente en la fila de la quebrado Obispo en San Ramón. Rellena vetillas en el tajo Dantas, asociado a las zeolitas fibrosas y abunda en varias formas y tonos en el tajo de Guaitil de Acosta. Sin embargo, es un mineral muy raro en Pedernal y en La Cruz.

$$
\text { Girolita } \mathrm{Ca}_{2} \mathrm{Si}_{2} \mathrm{O}_{4}(\mathrm{OH})_{2} * \mathrm{H}_{2} \mathrm{O}
$$

Cristales blanquecinos en grupos radiales de pocos milímetros de diámetro, asociada con las zeolitas del tajo La Garita. 
Malaquita $\mathrm{Cu}_{2}(\mathrm{OH}){ }_{2} \mathrm{CO}_{3}$

Característico es su color verde que se presenta en agregados musgosos, en costras y a veces botroidal acompañando a la laumontita en Cóncavas de Cartago.

Okenita $\mathrm{Ca}_{3} \mathrm{Si}_{6} \mathrm{O}_{12}(\mathrm{OH})_{6} * 3 \mathrm{H}_{2} \mathrm{O}$

Se presenta en cristales aciculares blancos, agregados en forma radial. Acompaña la estilbita y chabasita en Pedernal de Puriscal.

Prehnita $\mathrm{Ca}_{2} \mathrm{Al}_{2}(\mathrm{OH})_{2} \mathrm{Si}_{3} \mathrm{O}_{10}$

Se le encuentra en cavidades de hasta $1 \mathrm{~cm}$, formando agregados esféricos de cristales tabulares fuertemente estriados. Es transparente y translúcida con tonos de amarillo verdoso tenue, asociada con chabasita y analcima en el río Barbilla en Siquirres.

Todorokita $\left(\mathrm{Na}_{2} \mathrm{Ca}, \mathrm{K}_{2}\right) \mathrm{Mn}^{4+}{ }_{4} \mathrm{Mn}^{3+}{ }_{2} \mathrm{O}_{12} * 3 \mathrm{H}_{2} \mathrm{O}$
Es un mineral de brillo metálico, iridiscente con reflejos a la luz de colores azul, gris rojizo hasta casi negro. Solo se encontró en Pedernal de Puriscal, donde rellena cavidades microscópicas.

\section{CONCLUSIONES}

En los alrededores del Valle Central y en la zona de Siquirres se encontraron en 25 afloramientos, 19 zeolitas diferentes y 10 minerales asociados. Las zeolitas encontradas son: Analcima, natrolita/paranatrolita, gonardita, estilbita, mordenita/escolecita, epistilbita, gismodina, cowlesita, laumontita, tschernichita, heulandita, levinita, chabasita, erionita/offretita, thomsonita, garronita, filipsita, goosecreekita(?) y yugawaralita(?).

Las zeolitas se presentan dentro de vetillas y rellenando vesículas, principalmente en los basaltos y rocas piroclásticas de las formaciones La Cruz y Grifo Alto, y en los basaltos alcalinos e intrusiones hipoabisales de Guayacán. Las

Cuadro 1

Variedades de zeolitas en las localidades visitadas

\begin{tabular}{|c|c|c|c|c|c|c|c|c|c|c|c|c|c|c|c|c|c|c|c|c|c|}
\hline ZEOLITAS & 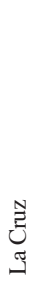 & 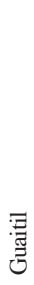 & 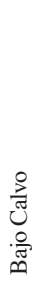 & $\frac{\frac{8}{0}}{0}$ & 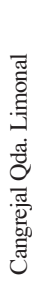 & \begin{tabular}{l}
$\frac{\circ}{2}$ \\
$\frac{0}{2}$ \\
\hdashline \\
\hdashline
\end{tabular} & 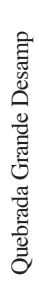 & 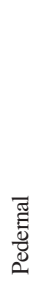 & 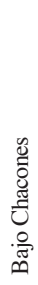 & 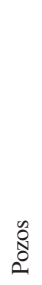 & 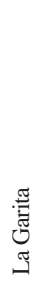 & 恚 & 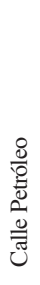 & 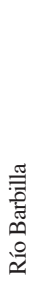 & 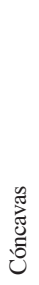 & 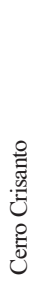 & 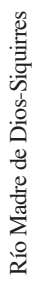 & 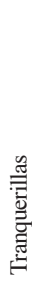 & 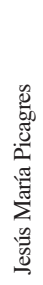 & 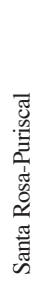 & 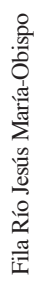 \\
\hline Analcima & $\mathrm{X}$ & & $\mathrm{x}$ & & & $\mathrm{x}$ & $\mathrm{x}$ & & & & & & & $\mathrm{x}$ & & & & & & & \\
\hline Chabasita & $\mathrm{x}$ & $\mathrm{x}$ & $\mathrm{x}$ & & & $\mathrm{x}$ & $\mathrm{X}$ & $\mathrm{X}$ & & & $\mathrm{X}$ & $\mathrm{X}$ & $\mathrm{x}$ & $\mathrm{x}$ & & $\mathrm{x}$ & $\mathrm{X}$ & $\mathrm{X}$ & $\mathrm{X}$ & & \\
\hline Cowlesita & $\mathrm{X}$ & & & & & & $\mathrm{X}$ & $\mathrm{X}$ & & & & & & & & & & & & & \\
\hline Epistilbita & & $\mathrm{x}$ & & & & & & $\mathrm{X}$ & & & & & & & & & & & & & \\
\hline Erionita/ Ofertita & & & & & & & & $\mathrm{X}$ & & & & $\mathrm{X}$ & $\mathrm{x}$ & $\mathrm{X}$ & & & & & & & \\
\hline Escolecita & & & & & & & & & $\mathrm{x}$ & $\mathrm{x}$ & & & & & & & & & & & \\
\hline Estilbita & $\mathrm{x}$ & $\mathrm{x}$ & & $\mathrm{x}$ & & $\mathrm{x}$ & & $\mathrm{x}$ & $\mathrm{x}$ & $\mathrm{x}$ & $\mathrm{x}$ & $\mathrm{x}$ & & & & $\mathrm{x}$ & & & & $\mathrm{x}$ & $\mathrm{x}$ \\
\hline Filipsita & & & $\mathrm{x}$ & & & $\mathrm{x}$ & $\mathrm{X}$ & $\mathrm{X}$ & & & & $\mathrm{X}$ & & $\mathrm{x}$ & & & & & & & \\
\hline Gismondina & & & & & & & & & & & & & & $\mathrm{x}$ & & & & & & & \\
\hline Gonardita & & & $\mathrm{x}$ & & & & & & & & & & & & & & & & & & \\
\hline Goosecrekita & & & & & & & & $\mathrm{X}$ & & & & & & & & & & & & & \\
\hline Heulandita & $\mathrm{x}$ & $\mathrm{x}$ & $\mathrm{x}$ & & & & $\mathrm{x}$ & $\mathrm{X}$ & & & & & & & & & & & & & \\
\hline Laumontita & $\mathrm{x}$ & $\mathrm{x}$ & $\mathrm{x}$ & $\mathrm{x}$ & $\mathrm{x}$ & & & & $\mathrm{x}$ & & & & & & $\mathrm{X}$ & & & $\mathrm{x}$ & & & $\mathrm{x}$ \\
\hline Levinita & $\mathrm{x}$ & & $\mathrm{x}$ & & & & $\mathrm{x}$ & $\mathrm{X}$ & & & & $\mathrm{x}$ & $\mathrm{X}$ & $\mathrm{x}$ & & & & & & & \\
\hline Mesolita/ Mordenita X & $\mathrm{x}$ & $\mathrm{x}$ & $\mathrm{x}$ & & & & $\mathrm{X}$ & $\mathrm{X}$ & & $\mathrm{X}$ & $\mathrm{X}$ & & & & & & & & & & \\
\hline Natrolita & & & & & & & & & & & & $\mathrm{X}$ & & $\mathrm{X}$ & & & & & & & \\
\hline Thomsonita & $\mathrm{x}$ & & $\mathrm{x}$ & & & $\mathrm{x}$ & $\mathrm{x}$ & $\mathrm{X}$ & & & & $\mathrm{x}$ & & $\mathrm{x}$ & & $\mathrm{x}$ & & & & & \\
\hline Tschernichita & & & & & & & & $\mathrm{X}$ & & & & & & & & & & & & & \\
\hline Yugawaralita & & $\mathrm{X}$ & & & & & & & & & & & & & & & & & & & \\
\hline
\end{tabular}


paredes de las cavidades están cubiertos generalmente por arcillas y sobre estas cristalizaron las zeolitas (Tschernich \& Boyd, 1993).

Las especies más frecuentes son chabasita, estilbita, laumontita, mesolita/mordenita, thomsonita y analcima, que abundan tanto en las rocas del Valle Central como de la zona de Siquirres (Cuadro 1). Otras especies, como la girolita, gismondina, gonardita, goosecrekita, tschernichita y yugawaralita son escasas y fueron encontradas en pequeña cantidad en un solo afloramiento. Muchas de estas zeolitas raras se encuentran en unas pocas vesículas de las rocas y faltan generalmente en vesículas adyacentes, las cuales solo contienen especies más comunes. La natrolita, que es una de las zeolitas más frecuentes en otras partes del mundo, solo se encontró en los alrededores de Siquirres.

Basado solamente en las observaciones macroscópicas y microscópicas, la clasificación de las zeolitas es difícil y no muy segura. La clasificación definitiva de las especies goosecrekita y yugawaralita solo se logrará con la búsqueda paciente y con suerte para encontrar una mayor cantidad de material que permitirá un análisis por difractometría de rayos-X.

\section{AGRADECIMIENTOS}

Se agradece a Don Ronald Boyd por su entusiasmo, paciencia y sus consejos y por abrirme los ojos a una visión nueva de la naturaleza de nuestro pequeño país. A mi hijo Néstor por las traducciones y a mi familia por darme ánimo en todos estos años y a don S. Kussmaul por la invitación a escribir este artículo.

\section{REFERENCIAS}

DANA E.S. \& FORD, E.N., 1973: Tratado de mineralogía. 850 págs. Ed. Continental, México.

HAY, R.L., 1978: Geologic occurrence of zeolites. - En: SAND, L.B. \& HUMPTON, F.A. (eds.): Natural Zeolitas: Occurence, properties, use. - Pergamon Press, Oxford: 135-143.

PÉREZ, W., 1996: Estudio geológico y amenazas naturales en el corredor Turrialba - Siquirres, Poliducto de RECOPE, Sector Laguna Bonilla - Guayacán. - 71 págs. Univ. de Costa Rica [Inf. Campaña Geol.].

TSCHERNICH, R., 1992: Zeolites of the World. - 526 págs. Geosciences Press.

TSCHERNICH, R., 1993a: Zeolites of Costa Rica. - Micro Probe VII(7): 3-9.

TSCHERNICH, R., 1993b: Epistilbite and associated minerals from Pedernal, Costa Rica. - Micro Probe, VII(7): 12-14.

TSCHERNICH, R. \& BOYD. R., 1993: Preliminary report of the second occurrence of tschernichite. - Micro Probe, VII(8): 4-6.

TOURNON, J., 1984: Magmatismes du Mésozoïque á l'Actuel en Amérique Centrale: l'exemple de Costa Rica, des ophiolites aux andésites. - 335 págs. Univ. P. Et M. Curie, Paris [Tesis PhD.]. 